\title{
An Innovative Approach to Recognize and Generate the Design Patterns as Formative Concepts to Support the Design Computation
}

\author{
Kulwant Singh \\ Saraf Institute of Engineering \& \\ Technology, Hanumangarh \\ Rajasthan, India
}

\author{
Atul Kumar \\ Saraf Institute of Engineering \& \\ Technology, Hanumangarh \\ Rajasthan, India
}

\author{
Nitin Sharma \\ Saraf Institute of Engineering \& \\ Technology, Hanumangarh \\ Rajasthan, India
}

\begin{abstract}
This work focuses on integrated product design and computation, and proposes methodologies and tools to build product designs integrating CAD-CAM technologies. The designs belonging to different eras are generated automatically with the help of developed algorithms and their fabrication methodologies, thus, helping the designer to reach his objective. Although most of the designs that are produced using this method, are non functional, the decorative aspect of the final product serves a great purpose when looked at in terms of the product's visual and emotional attractiveness to the end consumer. This is achieved through the use of different forms and patterns.
\end{abstract}

This proposed work presents different decorative design patterns as formative concepts to support the design computation. The design of any pattern in a particular artform, is identified by the physical shape of the primitives of the motifs/compound and motifs/patterns, as well as its attending spatial interactions. By using this little "trick," mathematical models or parametric modeling of the design patterns can thus be formulated. The variability and embeddedness in the design patterns can be explored by this methodology.

\section{Keywords}

CAD/CAM, Design, Motif, Pattern, Recognize.

\section{INTRODUCTION}

The tradition of exquisite decorative design patterns exhibits from the years in the history. The decorative design patterns are accepted in all applications of designing which belongs to different arts. The artistic design patterns can be use to decorate the screens, cabinets, doors, windows, temples and furniture in fashionable traditions. All design patterns are easily recognize and famous, for its uniqueness, attractiveness, meticulousness. Till now, the designer starts their innovative ideas of creativity in their profession of design with the help of paper and pencil that is passed on through generations. Due to its complex traditional designing and production with hand, many problems are arising on the future of such art form. Although, decorative designing is one of the growing traditions in the world, but, it is just growing in leaps and bounds. Therefore, it requires to be empowered with digital technology for making it globally sustainable [8]. Further in fact, globalization has added challenges for the local artist as they are now being forced to produce the better esthetics look with quality product in shorter time span and sell their products at reasonable prices. Thus, there is an urgent need for cost effective automated system for the designers and handicraft workers, so that these people would be able to reproduce the identical finished products economically and in smaller time duration. But, local artists in this part are also very unwilling to accept new technology because of fear to lose the heritage style of designs [13].

Obviously, computer aided designing saves time when compared to manual designing. So, several commercial CAD packages are available in the market in order to perform computer aided designing. But, these $\mathrm{CAD}$ packages are beyond the reach of the designers in terms of price. Moreover, operating these packages require a deep knowledge of CAD as subject, which is not easy to get by the artists. As a result, the use of these systems is being restricted. Rather, the designers who are designing through these commercial CAD systems, their skill is increasingly decreased as working in CAD systems cannot satisfy their instinct for drawing on paper. It also degrades designer's visual thinking and hampers his/her generative ability in conceptualizing abstract form. Therefore, there is need of a tool which offers the possibility for assisting craftsmen in creating computer aided decorative design patterns. For this reason, this work presents Innovative Methodologies and Tools to Build Product Designs Integrating CAD-CAM Technologies that will not only save the time but also provide opportunities to produce creative designs. Moreover, this concept will serve to explore decorative traditional patterns in different ways than hand drawing.

The knowledge of design learned and abstracted from existing artifacts plays vital roles in design measures. It serves as a source for the derivation of design, guide and controls the design processes and enables the designer and the computer to evaluate the results of creative designing with their innovative ideas. The aesthetic values can be evaluated using proportion and symmetry existing in the designs. A distinctive and repetitive work of art relationship between elements/primitives/ motifs/compound motifs is analyzed formally and regarded as a design pattern [11\&12]. It is an invariant in patterns that appears repeatedly in one compound motif or in a set of compound motifs.

Now, recognition of design knowledge from existing objects and its representation are important for supporting computerbased design algorithms/ procedures as well as for human design activity. The patterns can be recognized in terms of repetitions of similarities as well as the theme of the pattern [2]. A pattern, that is a repetition of motifs/compound motifs, such as a repetition of the same triangles, a repetition of 
rectangles, and a repetition of ovals, can be easily recognized using feature similarity. However, a pattern that is constructed from different low-level motifs in a complex motifs say compound motifs is not recognized in terms of feature similarity. Similarity of spatial interaction needs to be considered for design pattern recognition and representation. A pattern that is recognized and represented operationally is useful for design procedures such as style learning, analogy and complexity measure.

\section{LITERATURE}

By using the field of computer graphics, a lot of researches have taken place to produce a computer-generated decorative pattern with or without recognizing the pattern, which offers many algorithmic possibilities for creating two-dimensional decorative effects. Chua [1] described a method for generating parametric patterns aligned along a circular arc. Kaplan [2] presented a process for creating computer-generated Islamic star patterns. C.S. Wang [3] presents the effective method of digitizing Chinese Calligraphy font using CAD software. Peter R. Cromwell [4] produces new 3-fold designs in the Islamic style. Gulati [5-6] developed a parametric CAD modeler for creating star patterns to produce traditional piece of lattice-worked screens. Shrivastva [7] made an attempt to develop parametric traditional Rangoli and Phulkari patterns. Trivadi [8] presented an application of CAD for producing Moradabad brassware patterns. Wong [9] described and explored the ways in which floral patterns can be created algorithmically. Kedar [10] proposed a design software for generating 2D floral border patterns. Anderson [11] created beautiful ornamental 2D patterns, which follows a userdefined curve. Gulati [12-13] presented a hierarchic representation scheme for generating decorative pattern and presented a paradigm for generating motifs of Saharanpur art. Kulwant [14] described role of mathematics in designing the decorative patterns.

\section{PATTERN REPRESENTING}

\section{ASPECTS}

Adequacy of the representation is critically important, because the process of pattern representation can be regarded as a search for possible descriptions that the computer formulates from given input data. Firstly, the representation should describe the particular concept appropriately and it should have the ability to represent implicit as well as explicit knowledge. Secondly, it should have a well-organized form so that it can perform inferences correctly with predefined knowledge. Thirdly, from the result of pattern representations, new objects should be able to be predicted or generalized.

Decorative patterns are defined in terms of abstract entities called motifs which are neither overlapped nor separated at awkward distances with respect to pattern. These are based on the repetition of motifs positioned at regular, or measured, intervals. While generating decorative patterns, it is necessary to have a realization that how more complex patterns can be created from simpler shapes.

Throughout the history, humans have decorated fabrics, pottery vessels, tools and buildings with patterns. Repeated usage of the same motifs in decoration gives artifacts patterns. The craftsmen's understandings of motifs create good patterns. Natural orders surrounding us provide examples of patterns. Forms of plants are the most popular motifs in capitol designs, window designs and many other artifact designs. Patterns have many characteristics in design. They often summarize design knowledge which appears in the existing design. Patterns are generalized from a set of objects belonging to a particular class. Related patterns can be composed in a hierarchical form and layered. Patterns expressed in the form of hierarchical tree structures [12] have variability at their lower levels. The application of invariant high-level patterns can generate many possible results under different contexts.

\subsection{Pattern Design:}

A design is composed of motifs, and patterns may have relationships with each other. Designs are recognized explicitly and implicitly.

A pattern is a set of bounded poly lines design represented as follows:

Where,

$\mathrm{D}=\{\mathrm{M} 1, \mathrm{M} 2, \ldots, \mathrm{Mn}\}$

M: bounded poly lines design

D: Pattern Design object

There are many different patterns in terms of design properties: motif, compound motif and pattern.

\subsubsection{Motif}

A pattern can be decomposed into compound motifs. Compound motifs are subparts embedded in a design and they may have spatial relationships between each other labeled as motif,

\subsubsection{Compound motifs}

A pattern is composed of a set of compound motifs and their relationships. Relationships are compositional rules of design. Pattern that cannot be decomposed into compound motifs are primitive design, among these, squares, circles and equilateral triangles are regarded as pure primitive motifs.

\subsubsection{Pattern}

Compound motifs/motifs are grouped together visually in terms of their properties and relationships, such as proximity, similarity, closure, good continuation, symmetry. Congruent primitive motifs are grouped together and form a compound motif. Motifs, either compound motifs or pattern, can be embedded into other motifs. The pattern may be regarded as a primitive motif when a set of congruent compound motifs are grouped together to make a pattern. A pattern design object is composed of compound motifs and it is represented as follows:

$$
\begin{aligned}
& \mathrm{Ci}=\{\mathrm{Mi}, 1, \mathrm{Mi}, 2, \ldots, \mathrm{Mi}, \mathrm{n}\} \\
& \mathrm{D}=\{\mathrm{C} 1, \mathrm{C} 2, \ldots, \mathrm{Cn}\}
\end{aligned}
$$

Where

C: Compound motif

D: Pattern Design object 


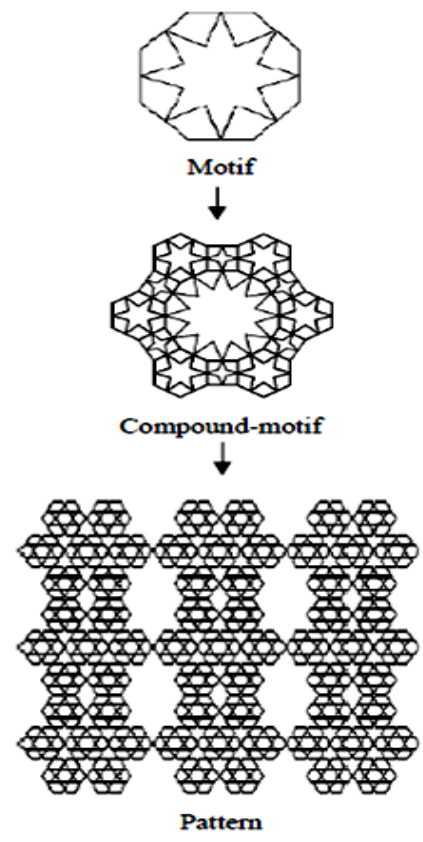

Fig 1: Hierarchical Representation of the Pattern

In this view, the basic idea of the representation scheme is to capture the symmetry amongst the patterns that are realized as a symmetric arrangement of similar or different shapes in planar map through the use of transformation rules that defines positions of shapes with respect to pattern in a twodimensional plane. In particular, patterns are understood as a set of shapes smash into parts as observed the symmetry of the pattern. This representation scheme has used CAD as a design tool for creating parametric motifs and arranging them into some definite order to produce a pattern.

This section presents an approach for parametric modeling of design motifs with use of computer algorithms in a programmable environment. All the patterns use the traditional motifs which are well-known for its unique style. In order to make possible the variation in a class of motif; parametric modeling has been performed through some the controlling parameters. The subsequent section is about the derivation of coordinates in terms of parameters for creating different shapes of petal/primitive related to different motifs of our study from all classes. The different patterns studied by us classified into various categories, like interlacing pattern, floral pattern, star pattern, border pattern, tessellation pattern etc (see figure 2)

\section{INVENTION OF A PATTERN}

In a pattern, motifs are placed with a special theme e.g. aligning motifs along a particular path or in a rectangular array. The geometric patterns are regulated by the formal rules of mathematical symmetry [5 \&14] which is recognized with transformation that leads to it. Patterns are composed of compound motifs/motifs which are multiplied and ordered in regular sequences by using transformations between them. A rectangular array of compound motifs/motif is generated using the following parameters: (1) Number of rows (R) (2) Number of columns (C) (3) Row offset (RO) (4) Column offset (CO). The first two parameters are taken according to the size of the surface of the pattern to be created. The row and column offsets parameters depend on the size of generated motif. From "Figure 3", the pattern is broken into motifs to simplify its structure. Further the motif is identified the repeated view of the primitive. Then the parametric modeling of the smaller entity labeled as primitive is essential to understand the view of motif and pattern.
(C)

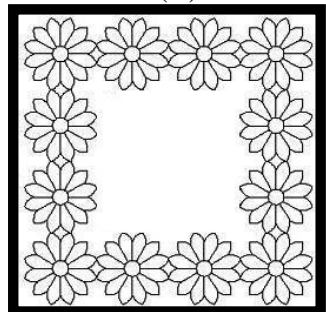

(E)

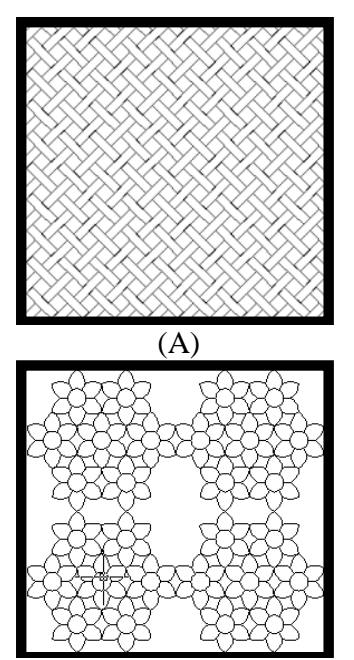

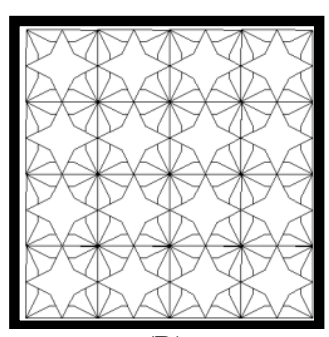

(B)

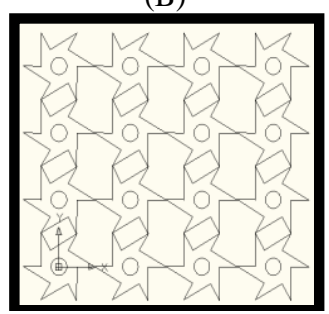

(D)

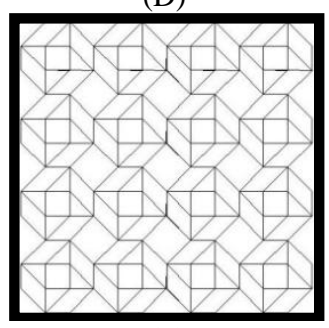

(F)
Fig 2: A) Interlaced pattern, B) Floral Pattern, C \& D) Star pattern, E) Border Pattern, F) Tessellation Pattern.

This motif has a special theme behind its shape. It is a double stared motif. This motif is drawn by using the primitive showing red. The primitive is drawn by eight lines which are $\mathrm{AB}, \mathrm{BC}, \mathrm{CD}, \mathrm{AD}, \mathrm{AF}, \mathrm{FH}, \mathrm{AE}$ and $\mathrm{EG}$. These eight lines have eight controlling points as shown in figure 3. In CAD modeling, this motif is seemed as an array of primitive which is an atomic geometric object made from 2D entities i.e. line. It has a set of points whose coordinates have been devised in terms of the following intuitive modeling parameters:

- Number of petals $(n=4)$ in the motif

- Size of the motif defined by its side ' $a$ '

Table 1: Coordinates of the points in the primitive of Motif

\begin{tabular}{|c|c|c|c|}
\hline Points & $\begin{array}{l}\text { Position of } \\
\text { the Points }\end{array}$ & Points & $\begin{array}{l}\text { Position of } \\
\text { the Points }\end{array}$ \\
\hline A & 0,0 & $\mathrm{E}$ & $\frac{a}{6}, \frac{a}{3}$ \\
\hline B & $0, \frac{a}{2}$ & $\mathrm{~F}$ & $\frac{a}{3}, \frac{a}{6}$ \\
\hline $\mathrm{C}$ & $\frac{a}{3}, \frac{a}{3}$ & $\mathrm{G}$ & $\frac{a}{6}, \frac{5 a}{12}$ \\
\hline $\mathrm{D}$ & $\frac{a}{2}, 0$ & $\mathrm{H}$ & $\frac{5 a}{12}, \frac{a}{6}$ \\
\hline
\end{tabular}




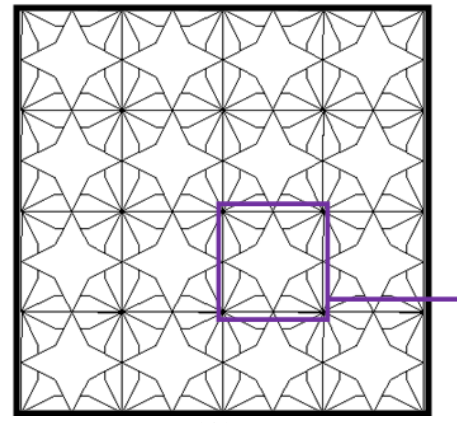

(A)

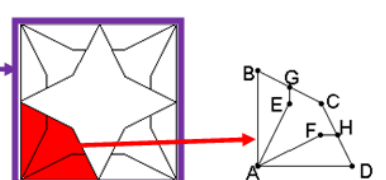

(B)

(C)
Fig 3: Geometry of Primitive of Motif; A) Pattern, B) Motif, C) Primitive.

This work has been implemented under the ActiveX and VBA programming environment using AutoCAD. VBA runs in the same process space as AutoCAD which provides programmable control through the ActiveX Automation interface. By putting the modeling parameters, the different decorative motif patterns can be generated by this CAD model. This work has been also validated by producing the patterns on a computer controlled laser cutting machine (see figure 4)

\section{APPLICATIONS OF PATTERN REPRESENTATION IN DESIGN}

The design pattern representation is fundamental in many areas in design computation. Understanding, interpretation and learning about design patterns are fundamental procedures for design computing. Recognition of pattern elements and their organization supports interpretation and learning processes. Recognition processes may identify only a part of design knowledge or superficial knowledge due to complexity of pattern design. Complex designs are made up of a large number of motifs/compound motifs that interact in a non-simple way. Motifs/compound motifs in a whole designed shape are not arranged by mere chance, but organized and ordered. Identification of those orders may cause a decrease of visual complexity. Complexity and simplicity of designs may be measured in terms of normalizing the length of design pattern descriptions. But an increase in order does not necessarily means a decrease in complexity. Measurement of the complexity would be useful in the evaluation of some form of aesthetics. Patterns as invariant knowledge help designers making decision in solving problems and can be adapted to new design situations with instantiations of some level variables. Patterns are sometimes expressed as rules of thumb in design. Recognition and representation of patterns from previous designs are important for designing.

\section{CONCLUDING REMARKS}

Based on the notion that the design is the application of previous design knowledge to a new design situation, design knowledge representation, particularly design pattern representation, has been investigated to support design computation. Representation of design knowledge can help the designer's understanding and interpretation, and the computer's use of design concepts of previous design works. Interpretations from appropriate design knowledge representations could support innovative and creative design.
Design pattern knowledge is being constructed in a hierarchical tree structure based on repetitions of similarities as well as congruency of motifs/compound motifs and interrelation between them.

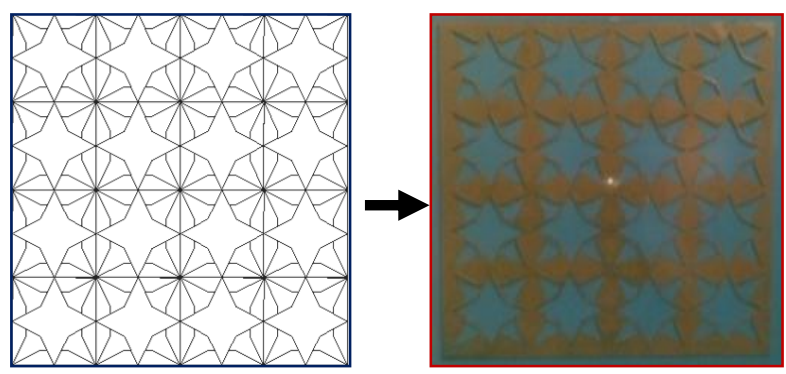

Fig 4: CAD Design model of floral pattern (Left) and Actual pattern cut by computer controlled laser cutting machine (Right)

\section{REFERENCES}

[1] Chee Kai Chua, Robert Gay and Wolfgang Hoheisel , "A Method of Generating Motifs Aligned Along a Circular Arc", Computer \& Graphics, Vol. 18, No. 3, pp. 353362, 1994.

[2] Kaplan C. S. "Computer Graphics and Geometric Ornamental Design", Ph.D., University of Washington, Seattle, 2002.

[3] Wang, C. -S., Chang, T. -R., Hsiao, C. -Y. and Teng, C. K.'Product development for Chinese calligraphy using reverse engineering and rapid prototyping', Virtual and Physical Prototyping, Vol. 1, No. 4, 259 - 269

[4] Cromwell, Peter R.(2010) 'Islamic geometric designs from the Topkap1 Scroll I: unusual arrangements of stars', Journal of Mathematics and the Arts, Vol. 4, No. 2 , $73-85$

[5] Vishal Gulati and Puneet Katyal, "Parameterized Modeling of Star Patterns for Traditional Latticed Screens”, International Journal of Computer Applications, Vol. 11- No.1, 2010.

[6] Vishal Gulati, Puneet Tandon, Hari Singh, "A CAD Paradigm to Produce Zillij Style of Geometrical Patterns for Wooden Carvings", International Journal of Computer Applications Vol. No.3, 2010.

[7] Rahul Shrivastva, "Customization of CAD Modelling Software Using Parametric Macros for Design of Machinable Artistic Surface Patterns", M-Tech thesis, Thaper University Patiala, India, 2009.

[8] Trivedi S., Tiwari A., Chatterjee A., Pathak V., Dhande S. G. and Chauhan D. S., "Application of CAD, Rapid Prototyping and Reverse Engineering in Handicrafts Sector - A Success Story", 9th International Conference on Engineering Education, July 2006.

[9] Michael T. Wong, Douglas E. Zongker and David H. Salesin, "Computer-Generated Floral Ornament", University of Washington.

[10] Kedar S. P.," Geometric Modeling of Patterns", Master's thesis, Department of Computer Science and Engineering, Indian Institute of Technology, Kanpur, India, 2009.

[11] Dustin Robert Anderson, "Two-dimensional Computergenerated Ornamentation Using a User-driven global 
planning strategy", Master of Science in Computer Science, Thesis, California Polytechnic State University, San Luis Obispo, 2007

[12] Vishal Gulati and Puneet Katyal," A Hierarchic Representation Scheme For Generating Decorative Pattern"s, International Journal of Current Research Vol. 3, Issue, 11, pp.186-189, October, 2011

[13] Vishal Gulati, Kulwant Singh, Puneet Katyal, "A CAD Paradigm for Generating Woodworking Motifs", International Journal of Computer Applications) Volume 47- No.5, pp 38-40, June 2012

[14] Kulwant Singh, Atul Kumar, Nitin Sharma "The Mathematical Aspects in Art to Create Decorative Effect in Design Patterns", International Journal of Innovative Technology and Exploring Engineering, Volume-2, Issue-2, pp 36-39, January 2013

[15] Meili Wang, Jian Chang, Jian J Zhang." A Review of Digital Relief Generation Techniques", IEEE 2nd
International Conference on Computer Engineering and Technology, Vol.4, pp 198 -202, 2010

[16] A. van der Zee, B. de Vries, "Design by Computation" GA2008, 11th Generative Art Conference, pp 35-52

[17] Ronald Strebelow, Mirco Tribastoney, Christian Prehofer "Performance Modeling of Design Patterns for Distributed Computation"

[18] Grünbaum B. and Shephard G. C., 1992, Interlace Patterns in Islamic and Moorish art, Leonardo, 25, 331339.

[19] Dewdney A.K., 1993, The Tinkertoy Computer and Other Machinations, 222-230.

[20] George Bain, "The Methods of Construction of Celtic Art”, Dover, 1973. 